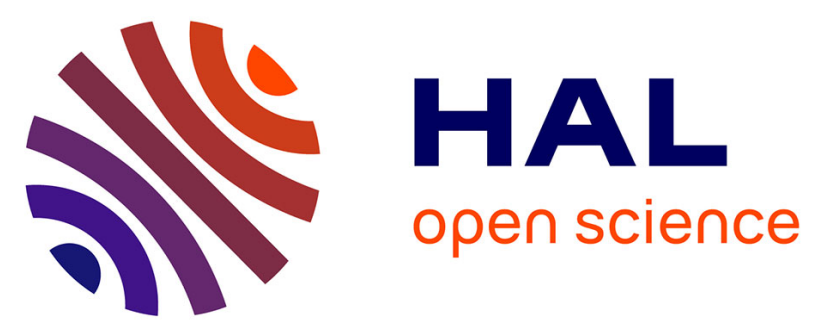

\title{
Top-coats for scalable nano-manufacturing with high- $\chi$ block copolymers in lithographic applications
}

\author{
Xavier Chevalier, Cindy Gomes Correia, Gwenaelle Pound-Lana, Philippe \\ Bézard, Matthieu Sérégé, Camille Petit-Etienne, Guillaume Gay, Gilles \\ Cunge, Benjamin Cabannes-Boué, Célia Nicolet, et al.
}

\section{To cite this version:}

Xavier Chevalier, Cindy Gomes Correia, Gwenaelle Pound-Lana, Philippe Bézard, Matthieu Sérégé, et al.. Top-coats for scalable nano-manufacturing with high- $\chi$ block copolymers in lithographic applications. SPIE Advanced Lithography conference - Alternative Lithographic Technologies, Feb 2021, San Jose, USA, France. pp.26, 10.1117/12.2583717 . hal-03452942

\section{HAL Id: hal-03452942 \\ https://hal.science/hal-03452942}

Submitted on 13 Dec 2021

HAL is a multi-disciplinary open access archive for the deposit and dissemination of scientific research documents, whether they are published or not. The documents may come from teaching and research institutions in France or abroad, or from public or private research centers.
L'archive ouverte pluridisciplinaire HAL, est destinée au dépôt et à la diffusion de documents scientifiques de niveau recherche, publiés ou non, émanant des établissements d'enseignement et de recherche français ou étrangers, des laboratoires publics ou privés. 


\title{
Top-coats for scalable Nano-manufacturing with High- $\chi$ Block Copolymers in Lithographic Applications
}

\author{
Xavier Chevalier ${ }^{1}, *$, Cindy Gomes Correia ${ }^{2}$, Gwenaelle Pound-Lana ${ }^{3}$, Philippe \\ Bézard $^{3,4}$, Matthieu Sérégé 3 , Camille Petit-Etienne ${ }^{3}$, Guillaume Gay ${ }^{3}$, Gilles Cunge ${ }^{3}$, \\ Benjamin Cabannes-Boué ${ }^{2}$, Célia Nicolet ${ }^{1}$, Christophe Navarro ${ }^{1}$, Ian Cayrefourcq ${ }^{1,5}$, \\ Marcus Müller ${ }^{6}$, Georges Hadziioannou ${ }^{2}$, Ilias Iliopoulos ${ }^{7}$, Guillaume Fleury ${ }^{2}$ and \\ Marc Zelsmann ${ }^{3}$
}

\author{
${ }^{1}$ ARKEMA FRANCE, GRL, Route Nationale 117, BP34 64170 Lacq, France. \\ ${ }^{2}$ Univ. Bordeaux, CNRS, Bordeaux INP, LCPO, UMR 5629, F-33600, Pessac, France. \\ ${ }^{3}$ Univ. Grenoble Alpes, CNRS, CEA/LETI Minatec, Grenoble INP, LTM, 38000 Grenoble, \\ France. \\ ${ }^{4}$ present address: IMEC, Kapeldreef 75, 3001 Leuven, Belgium. \\ ${ }^{5}$ present address: I-TEN SA, 12 Chemin du Jubin, F69570, Dardilly, France. \\ ${ }^{6}$ Georg-August Universität Göttingen, Institute for Theoretical Physics, 37077 Göttingen, \\ Germany. \\ ${ }^{7}$ Laboratoire PIMM, Arts et Métiers Institute of Technology, CNRS, Cnam, HESAM \\ Université, 151 Boulevard de l'Hôpital, 75013 Paris, France.
}

E-mail: xavier.chevalier@arkema.com

\begin{abstract}
Results for the self-assembly of lamellar silicon-containing high- $\chi$ BCPs with innovative neutral top-coat design are presented. We demonstrate that these materials and associated processes are compatible with a standard lithographic process, and oriented toward a potential HVM. We show that this dedicated technology is able to guarantee the stability and planarity of the stack even at elevated self-assembly bake temperatures, and opens new opportunity in the fields of 3D BCPs stacks. Finally, we show interesting results for the etch-transfer of a lamellar BCP in silicon.
\end{abstract}

\section{Introduction}

The directed self-assembly (DSA) of block-copolymers (BCPs) is a powerful method for the manufacturing of high-resolution features in lithographic applications. Studies performed over recent years have demonstrated impressive potential in terms of integration capabilities and defect levels with the well-known PS- $b$-PMMA system enabling its use within standard lithographic processes at an industrial level. However, this particular system is limited to dimensions above $\sim 20 \mathrm{~nm}$ in period, due to a rather weak segregation strength (measured by the Flory-Huggins parameter $\chi$ ) between both blocks, and new challenges have arised with the use of high- $\chi$ systems. Among them, rapid self-assembly capabilities, etch performances, integration flows, coatings uniformity, etc... are still major concerns and hardly addressed topics in existing literature, even though of crucial importance for nanomanufacturing with DSA. Early works on high- $\chi$ systems demonstrated some interesting potential with the use of top-coats (TC) to drive the perpendicular orientation of the BCP domains, ${ }^{1,2}$ but improvements are still needed for a realistic industrial implementation. For instance, wet-removal of TC in (1) 
may lead to defects and inhomogeneities across the wafer, and the TC coating may not be homogeneous either as the water-based solvent may not wet properly the $\mathrm{BCP}$ film due to surface tension mismatch. In (2), the TC process followed is appealing, but necessitates a PECVD-dedicated tool and an extended deposition time (>75 minutes) for the TC. Additionally, in this specific publication, the BCP cannot be transferred directly into the substrate and necessitate an additional time-consuming sequential infiltration process, precluding thus high-throughput of wafers.

In the present communication, we highlight our recent achievements on the design of specific top-coat materials used here with a silicon-containing high- $\chi$ BCPs systems based on poly(1,1-dimethyl silacyclobutane)-block-poly(styrene) (abbreviated PDMSB- $b$-PS), tackling successfully the aforementioned bottlenecks.

\section{Experimental}

Synthesis of TC material: the synthesis of the TC material was performed by radical polymerization in solution with AIBN as initiator. All the methacrylic monomers, solvent, initiator and terminating agent were used as received.

Synthesis of PDMSB-b-PS BCP: the synthesis of the BCP material was performed as previously reported. ${ }^{3}$

Preparation of thin films: NL material is dissolved into MIBK at 2\%, spin-coated onto $\mathrm{Si}$ monitor samples at $1500 \mathrm{rpm}$, grafted at $200^{\circ} \mathrm{C}$ during $75 \mathrm{sec}$, and then the samples are rinsed into MIBK. The $0.9 \mathrm{wt} \%$ PDMSB- $b$-PS solutions in MIBK were spin-coated $(2 \mathrm{krpm})$ on immobilized NLs followed by a soft-bake at $60^{\circ} \mathrm{C}$ for $1 \mathrm{~min}$ to produce $\mathrm{BCP}$ layers with approximatively $30 \mathrm{~nm}$ thickness. The TC material, previously dissolved into absolute ethanol at $2 \mathrm{wt} \%$, is spin-coated on the BCP thin film at $1500 \mathrm{rpm}$. The stack is baked at $90^{\circ} \mathrm{C}$ for 3 minutes, and the BCP is self-assembled at $240^{\circ} \mathrm{C}$ for 5 minutes.

Ellipsometry: The coating uniformity of the various polymeric layers was probed using spectroscopic ellipsometry with a phase modulated spectroscopic ellipsometer (UVISEL, from Horiba Scientifc) on the spectral range $200-800 \mathrm{~nm}$.

Scanning electron microscopy and scanning transmission electron microscopy: Top-view SEM images were acquired on a Hitachi CD-SEM H-9300 microscope with $0.5 \mathrm{kV}$ electron acceleration voltage and a $6 \mu \mathrm{A}$ current. STEM cross-sections were prepared and obtained with a Helios 450S-FEI dual-beam microscope.

Plasma etching: Etching experiments were performed in a $300 \mathrm{~mm}$ AdvantEdge ${ }^{\mathrm{TM}} \mathrm{MESA}^{\mathrm{TM}}$ from Applied Materials. In this inductively coupled plasma (ICP) etch tool the chamber walls are made of yttrium oxide. BCP samples were patched on $300 \mathrm{~mm} \mathrm{Si}$ wafers. The chamber was first cleaned using a three-step plasma: $\mathrm{NF}_{3} / \mathrm{Cl}_{2}$ for $45 \mathrm{~s}, \mathrm{Cl}_{2} / \mathrm{O}_{2} / \mathrm{Ar}$ for $45 \mathrm{~s}$, and finally $\mathrm{O}_{2}$ for $30 \mathrm{~s}$, to allow a good process reproducibility.

\section{Results and discussion}

The PDMSB- $b$-PS BCP system chosen here is depicted on the Figure 1. This system enables the formation of very high-resolution dimensions, as highlighted by the captured CDSEM images of lamellar systems shown in the Figure 1 presenting periods from 12 to $18 \mathrm{~nm}$. 
We note that further lowering of the dimensions could be still possible for the PDMSB- $b$-PS system, as the smallest lamellar morphology is well phase-separated even at the annealing temperature used here $\left(240^{\circ} \mathrm{C}\right)$. Such high- $\chi$ system is therefore quite appealing in the context of lithography with DSA. Moreover, the silicon-containing PDMSB block provides an additional etch-resistance for the resulting features, and thus avoid the use of time-consuming processes like sequential infiltration, to optimize the throughput of future related devices.

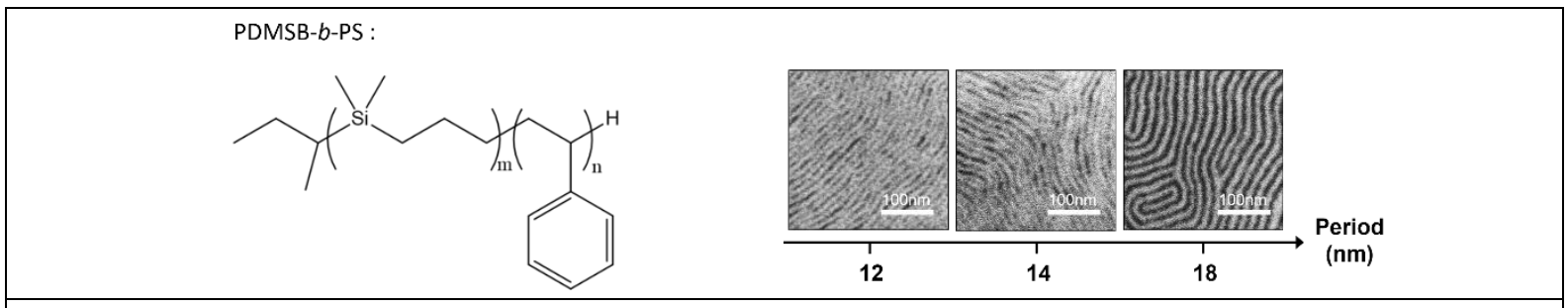

Figure 1: Chemical formula of PDMSB- $b$-PS system (left) and lamellar self-assembly obtained with the TC material for BCP periods down to $12 \mathrm{~nm}$ (right).

The whole set of materials used in the framework of the self-assembly is available in large amounts, as shown in Figure 2 where 1L bottles at $2 \mathrm{wt} \%$ of both the neutral layer (NL) and the TC, and $0.5 \mathrm{~L}$ for the $\mathrm{BCP}$, are displayed. The NL and TC materials are both based on a methacrylate chemical platform, and thus can be synthesized through a simple classical radical polymerization in high yields and purity over a wide range of compositions, and more importantly, on a scale of several $10^{\text {th }}$ of $\mathrm{kg}$ per batch if necessary. It can be noted that the TC itself can be used as a NL, as it contains chemical functions to enable the grafting of the material onto a variety of surfaces. These materials are not developed especially for the PDMSB- $b$-PS system, as they do not contain any of the BCP monomers in their formula, their respective composition is just easily adapted to this system to obtain a neutral material for the BCP. Therefore, these NL and TC materials can fit other BCP systems by a simple compositional adjustment if necessary while keeping intact their own intrinsic characteristics. The three materials involved in the self-assembly (NL, BCP, TC) are all able to be readily spin coated from the initial solution. The TC material is designed on purpose to be entirely soluble into ecofriendly solvents like alcohols (ethanol, IPA...), so as not to dissolve the BCP film during the spin-coating step. The respective process of the three materials, of a few minutes each before being entirely functional, is especially designed to favor a high throughput of wafer in the view of a potential HVM (Figure 2).

\begin{tabular}{|c|c|c|c|}
\hline & Neutral underlayer & BCP & Neutral TC \\
\hline $\begin{array}{c}\text { Film } \\
\text { Thickness }\end{array}$ & $\sim 2-5 \mathrm{~nm}$ & $\begin{array}{c}30 \mathrm{~nm}(\mathrm{std}) \\
1 \text {-many } \mathrm{L}_{0}\end{array}$ & $\begin{array}{c}50 \mathrm{~nm}(\mathrm{std}) \\
15-200 \mathrm{~nm}\end{array}$ \\
\hline Solvent & MIBK/PGMEA & MIBK & Alcohol (EtOh-IPA) \\
\hline Coating & Spin-coating & Spin-coating & Spin-coating \\
\hline $\begin{array}{c}\text { Processing } \\
\text { Time }\end{array}$ & $75 \mathrm{~s} / 2 \mathrm{mins}$ & $30 \mathrm{~s}$-hours & $\begin{array}{c}3 \mathrm{~min}(\mathrm{std}) \\
1-10 \mathrm{mins}\end{array}$ \\
\hline
\end{tabular}


Figure 2: Details of process parameters for the various materials involved in the selfassembly, showing compatibility with standard techniques and devices of lithography industry (left). Commercial bottles of $1 \mathrm{~L}, 0.5 \mathrm{~L}$ and $1 \mathrm{~L}$ for the NL, BCP and TC reported in this communication (right).

A special attention has been paid to the thickness uniformity for each layer involved in the final stack, as important variations are precluding any use of the materials into a potential application. The measurement reported here is performed on 4 inches wafers, with handdispensed materials for the corresponding spin-coated layers. A first visual inspection enables to confirm an excellent coating quality without any noticeable comet or other coating-related defects. Thicknesses variations were estimated through ellipsometry for each layer, from a series of automated measurements involving 31 independent points for each layer. The results reported in the Figure 3 further confirmed the excellent coating quality for each layer, with a $3 \sigma$ variation of $0.2,0.5$ and $2.4 \mathrm{~nm}$ for NL, BCP and TC materials, respectively. It is noteworthy that the TC uniformity of $2.4 \mathrm{~nm}$ is still less than $5 \%$ of the corresponding thickness across the whole wafer and cumulates the variation of the two other buried layers (NL and BCP). Therefore, the actual thickness variation of the TC layer itself is below this overestimated value, even after the self-assembly step performed at $240^{\circ} \mathrm{C}$ during 5 minutes.

\begin{tabular}{|c|c|c|c|}
\hline Material & Conditions & Thickness $(\mathbf{n m})$ & $\mathbf{3 \sigma}$-error $(\mathbf{n m})$ \\
\hline Neutral layer & Grafted and rinsed & 5.4 & 0.2 \\
\hline BCP Lam. 18nm & Coating + PAB & 33.4 & $\mathbf{0 . 5}$ \\
\hline Top-coat & $\begin{array}{c}\text { Coating+ self- } \\
\text { assembly }\end{array}$ & 50.4 & $\mathbf{2 . 4}$ \\
\hline
\end{tabular}



Figure 3: Thickness variations observed in 4 inches wafers of the different materials involved in the stack for the self-assembly process, showing a good coating uniformity for all the layers, even after the self-assembly annealing step performed ; 31 points of measurement performed for each layer (left). Ellipsometry map of the 31 measurements points highlighting the good uniformity of the TC layer (right).

The top-view SEM pictures for the various lamellar BCPs presented in the Figure 1 are already providing a good insight into the good quality of the self-assembly for each system. Indeed, upon selective removal of the TC through dry etch after the annealing step, well defined lamellar morphologies were observed on each random location probed by SEM. To gain further insights on the quality of the self-assembly, a series of FIB-STEM experiments were performed on the lamellar self-assembled films of $18 \mathrm{~nm}$ and $14 \mathrm{~nm}$ period, without the TC-etch step. The cross-sections obtained for these samples are presented in the Figure 4. For both systems, the STEM images show that the out-of-plane lamellar features are spanning the whole BCP film thickness $(30 \mathrm{~nm}$ for the $18 \mathrm{~nm}$ period and $40 \mathrm{~nm}$ for the $14 \mathrm{~nm}$ period, respectively), leading to high aspect-ratio features ( $\mathrm{AR}=\sim 6$ in the case of the $\mathrm{BCP}$ with $14 \mathrm{~nm}$ period). It confirms thus the well-balanced interfacial interactions for both blocks of the BCP with the TC and NL 
materials. We can note also that each interfaces involved in the system is well identified and separated from each other, confirming the absence of intermixing of the TC material inside the BCP layer.

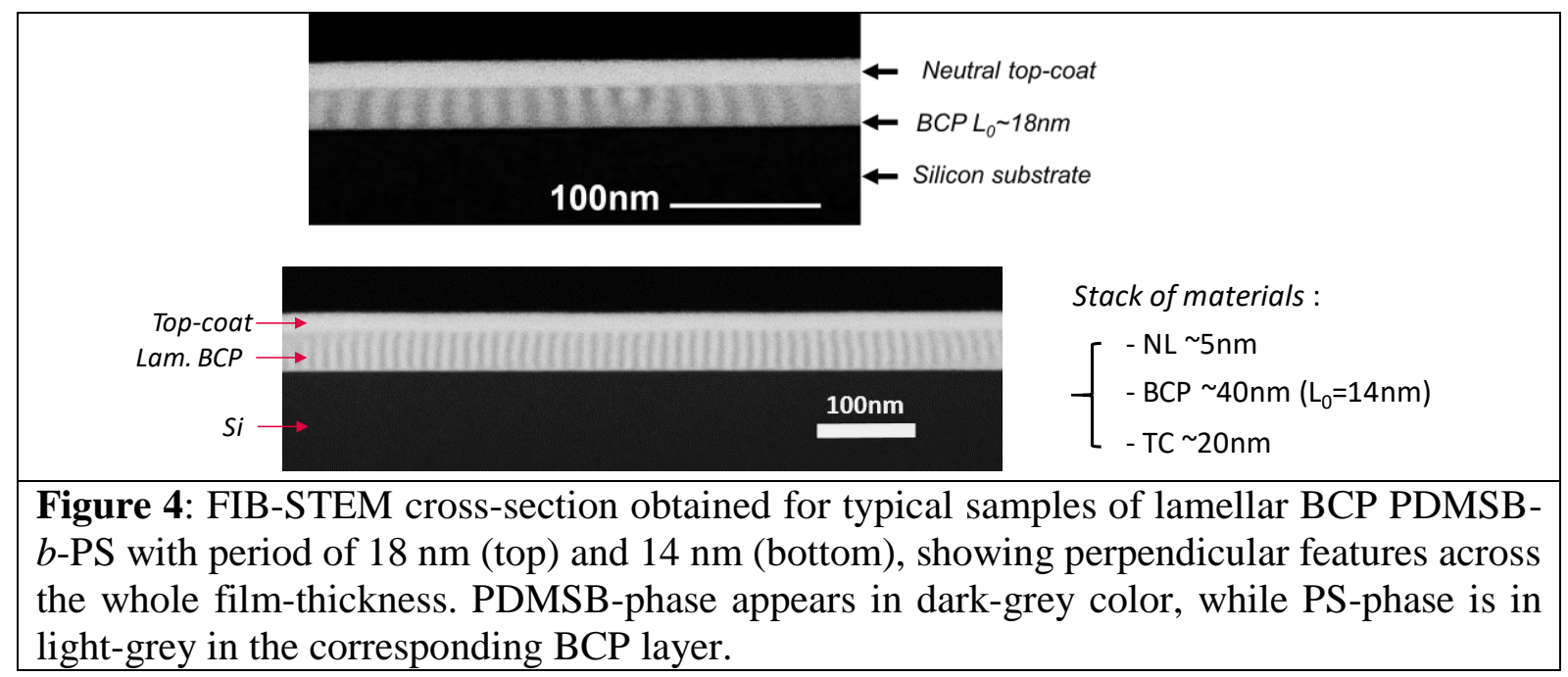

A unique aspect of these TC material is their propensity to guarantee the initial flatness of the stack despite of the various annealing treatments. Indeed, due to the particular configuration of DSA-usable materials (thin film, neutral interfaces), the well-known issue of dewetting is often present in BCP self-assembly, especially in the case of high- $\chi$ BCPs where the low molecular-weight and the large surface-energy difference between the blocks of the $\mathrm{BCP}$ favor dewetted configurations. The TC material designed in this study enables the circumvention of this critical issue owing to its particular macromolecular design. This exceptional propriety is demonstrated in the Figure 5, where the mean-area covered by the BCP layer $(18 \mathrm{~nm}$ period) for the inspected surfaces is plotted versus the self-assembly temperature of the system. One point on the graph correspond to one-dedicated sample made at the specified self-assembly temperature. From this graph, it is clearly observed that the dewetting start to proceed at $90^{\circ} \mathrm{C}$ for the standard neutral coating, leading quickly to an entirely dewetted configuration corresponding to a low BCP-covered area ( $10 \%$ only of the total inspected surface is covered by the BCP in the final state). Conversely, the BCP film coated by Arkema's neutral TC keeps its integrity and flatness even for high self-assembly temperatures $\left(250^{\circ} \mathrm{C}\right)$, as $100 \%$ of the inspected area is covered by the BCP. This technology not only ensures a fully functional self-assembled BCP thin film, but also enables to tremendously increase the thermal annealing budget for the self-assembly process in order to kinetically favor a potential defectfree configuration for a given BCP system. 


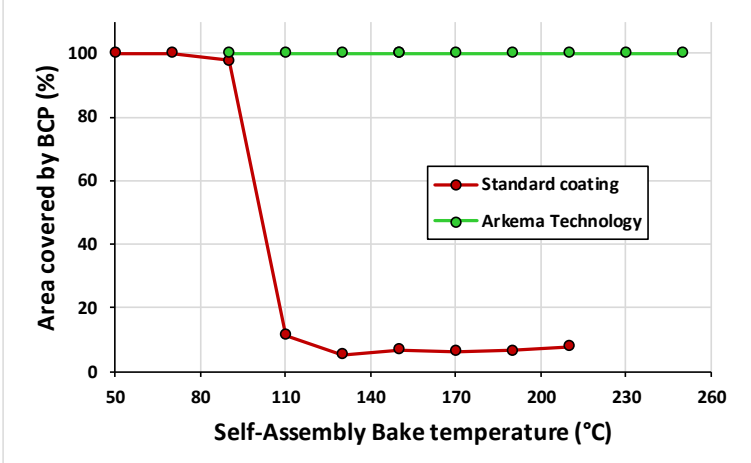

Figure 5: Dewetting experiment comparing Arkema's TC technology with a standard neutral TC. The standard coating does not prevent the dewetting of the BCP, while the Arkema's dedicated chemistry one is able to maintain the planarity of the stack even at very high selfassembly temperatures.

Another unique property of our TC technology dedicated to BCP self-assembly lies in the possibility of stacking various self-assembled BCP layers directly on top of each other. Such 3D-multilayered structures are potentially valuable in cutting-edge technologies as photonics, metamaterials and microelectronics through the extension of non-natural features obtained by BCP self-assembly. Although already reported in the literature, these multilayers stacks often necessitate additional processing steps to reinforce the buried layers as regards to a potential resolubilization along with the coating of new layers. These additional processes are somehow time-consuming, and lead to a significant decrease of throughput for the overall process. In the case of Arkema's technology, such additional processing steps are not necessary owing to the well-chosen constituents of the TC material itself. It results in a very swift process, as an entirely functional bilayer of BCPs such as the one depicted in the Figure 6 can be realized within less than 10 minutes (including a self-assembly bake of 5 minutes). Such a quick process, together with the multiple possible combinations to tune the final properties of the stack (orientation, morphologies, compositions, etc... see left panel of Figure 6), and the intrinsic readiness for the preparation of the stack, render these TC materials extremely appealing in view of such applications.

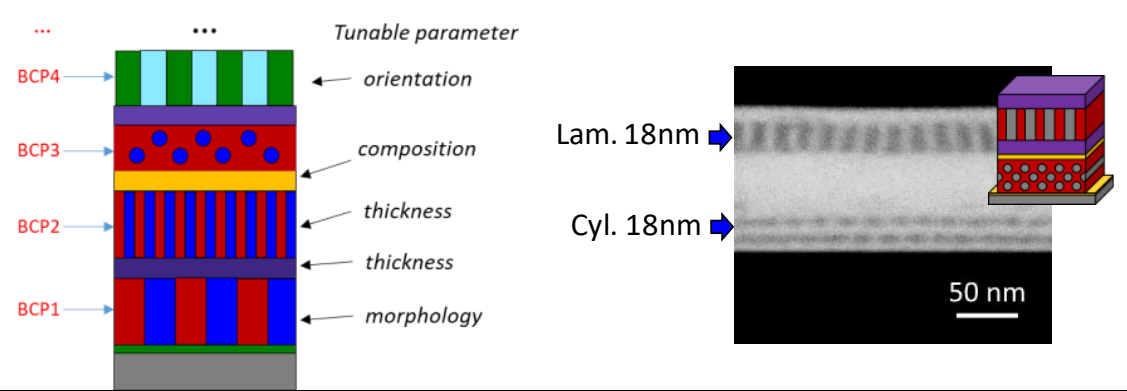

Figure 6: Potential tunable parameters in an hypothetical stack of BCPs to obtain different final properties (left). Example of stack of cylindrical BCP buried underneath a perpendicular lamellar BCP owing to Arkema's TC technology, enabling the stack to be ready in less than 10 minutes (self-assembly bake performed at $240^{\circ} \mathrm{C}-5$ minutes) (right).

The demonstration of the designed TC material as an optimal neutral layer for a siliconcontaining BCP is further corroborated through etch results. Indeed, the TC material can be readily removed with an $\mathrm{O}_{2}$-based plasma, owing to its fully organic nature, while the $\mathrm{BCP}$ 
layer behaves as an etch-stop layer for the very same plasma chemistry. The end of the TC-etch step is therefore quite straightforward with such materials combination. Afterwards, the PS phase can be removed selectively as regard to the PDMSB one, once again with an $\mathrm{O}_{2}$-based plasma, leaving at the end of the process low roughness silica-like perpendicular lamellae (Figure 7, left panel). A common patterning strategy is to then transfer the DSA patterns into a thin (5-10 nm, usually) amorphous Silicon hard mask ${ }^{8}$ to be used for further patterning steps, depending of the considered stack. Using synchronously-pulsed $\mathrm{HBr} / \mathrm{O}_{2}$ plasma, we demonstrate here that such oxidized PDMSB masks are resilient enough to pattern up to $45 \mathrm{~nm}$ deep features of CD $<9 \mathrm{~nm}$ in mono-crystalline Silicon and would therefore not be limiting in terms of hard-mask strategies (thickness, etc.) for any application.

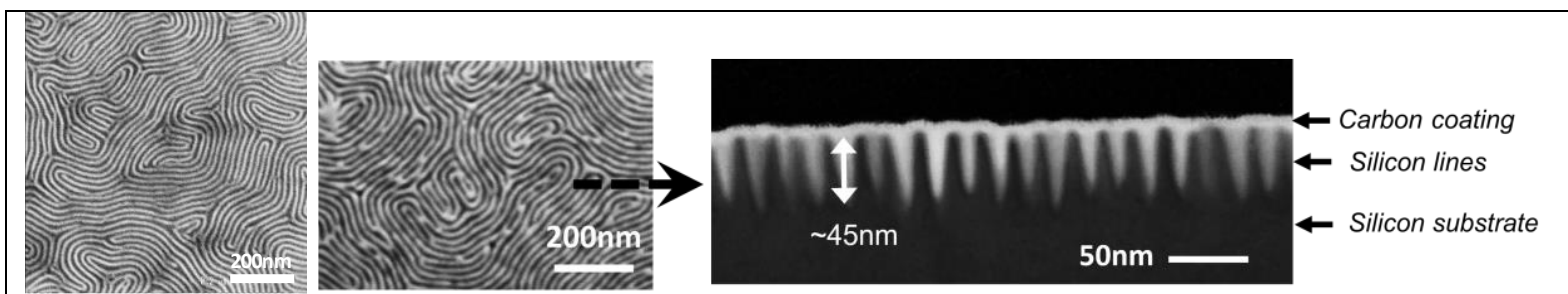

Figure 7: Top-view CD-SEM image of the PDMSB-b-PS lamellar BCP of $18 \mathrm{~nm}$ period, after PS selective removal with an O2-based plasma, showing a low roughness for the resulting mask (left). Top-view SEM image of the mask after transfer into the silicon substrate, showing a high-fidelity transfer from the initial mask (middle). FIB-STEM crosssection image of the transfer, showing $45 \mathrm{~nm}$-deep resulting fins-like features in silicon (right).

\section{Conclusions}

We have presented here the results relative to newly designed TC materials for BCP self-assembly with high- $\chi$ copolymers, taking into account well-known issues in DSA, and enabling a potential HVM for such processes. The optimized combination of these materials with a silicon-containing $\mathrm{BCP}$ enables the formation of high aspect-ratio perpendicular features, while allowing one to design functional stacks of BCP materials for advanced applications.

\section{References:}

(1) Bates, C. M.; Seshimo, T.; Maher, M. J.; Durand, W. J.; Cushen, J. D.; Dean, L. M.; Blachut, G.; Ellison, C. J.; Willson, C. G. Polarity-Switching Top Coats Enable Orientation of Sub-10-Nm Block Copolymer Domains. Science 2012, 338 (6108), 775779.

(2) Suh, H. S.; Kim, D. H.; Moni, P.; Xiong, S.; Ocola, L. E.; Zaluzec, N. J.; Gleason, K. K.; Nealey, P. F. Sub-10-Nm Patterning via Directed Self-Assembly of Block Copolymer Films with a Vapour-Phase Deposited Topcoat. Nat. Nanotechnol. 2017, 12 (6), 575-581.

(3) Aissou, K.; Mumtaz, M.; Portale, G.; Brochon, C.; Cloutet, E.; Fleury, G.; Hadziioannou, G. Templated Sub-100-nm-Thick Double-Gyroid Structure from SiContaining Block Copolymer Thin Films. Small 2017, 13, 1603777.

(4) Stefik, M.; Guldin, S.; Vignolini, S.; Wiesner, U.; Steiner, U. Block Copolymer SelfAssembly for Nanophotonics. Chem. Soc. Rev. 2015, 44 (15), 5076-5091. 
(5) Ross, C. A.; Berggren, K. K.; Cheng, J. Y.; Jung, Y. S.; Chang, J. B. Three-Dimensional Nanofabrication by Block Copolymer Self-Assembly. Adv. Mater. 2014, 26 (25), 43864396.

(6) Rahman, A.; Majewski, P. W.; Doerk, G.; Black, C. T.; Yager, K. G. Non-Native ThreeDimensional Block Copolymer Morphologies. Nat. Commun. 2016, 7 (1), 13988.

(7) Girardot, C.; Böhme, S.; Archambault, S.; Salaün, M.; Latu-Romain, E.; Cunge, G.; Joubert, O.; Zelsmann, M. Pulsed Transfer Etching of PS-PDMS Block Copolymers Self-Assembled in 193 Nm Lithography Stacks. ACS Appl. Mater. Interfaces 2014, 6 (18), 16276-16282

(8) Insert one of the IMEC's papers from Pathangi or from PESM, showing the stack used. 